\title{
Produção de pesquisa acadêmica internacional sobre gestão de recursos hídricos
}

A importância, escassez e gerenciamento da água movimentam continuamente pesquisas e debates acalorados. Diversas áreas de conhecimento dirimem esforços no sentido de diagnosticar as questões sobre o tema e propor soluções que permitam equacionar os conflitos inerentes. Nesse contexto, o objetivo deste artigo é analisar a produção de pesquisa acadêmica internacional sobre Gestão de Recursos Hídricos. Para tanto, realizou-se um estudo bibliométrico em que se lançou mão de técnicas avaliativas (medidas de produtividade e métricas de impacto) e técnicas relacionais (coautoria e coocorrência). Dessa forma, 358 publicações foram selecionadas e analisadas, utilizando-se como base a Web of Science. Os resultados descreveram as características da produção internacional - a exemplo da evolução da produção internacional, e países e publicações que são consideradas de impacto - assim como analisam a relação e formação de clusters de pesquisa via autores, países e organizações, indicando a composição de subtemas de estudo no campo de pesquisa escolhido para o estudo. $\mathrm{O}$ fator $\mathrm{H}$ ( $\mathrm{H}$-index) aponta para 41 publicações que podem ser consideradas referência na área. Observou-se um salto de publicações para o patamar atual em 2014 . Publicações norte-americanas e chinesas representam quase $40 \%$ do total. Constatou-se a existência de 12 clusters de termos, contendo 126 de maior relevância.

\section{Production of international academic research on water resources management}

\begin{abstract}
Water importance, scarcity and management constitute a continuous and intense debate on scientific research. Several knowledge circles direct efforts to understanding such related issues and take part on solutions to associated conflicts. The main goal pursued within this working paper is an analytical visualization of world scientific research on Water Resources Management. The technical orientation and procedures method approach intended to be a bibliometry, carried out using evaluative (productivity assessment and metrics) and relational (co-authorship and co-occurrence) techniques. 358 publications were selected and processed, having Web of Science as the chosen knowledge resource stream. Results construct a description of world production - such as its evolution, countries and publications, considering impact measures - as well as an analysis of relationship and established structure of research clusters by authors, countries and organizations, pointing to a composition of subordinated themes within the research field. The $\mathrm{H}$ factor ( $\mathrm{H}$-index) points to 41 publications that can be considered as area reference. 2014 was the stable achievement point of current publications levels. North American and Chinese publications represent almost $40 \%$ of the total production. It was found the existence of 12 terminological research clusters aggregated from 126 terms of greater relevance.
\end{abstract}

Keywords: Water Resources Management; Bibliometrics; World Scientific Production.

Topic: Desenvolvimento, Sustentabilidade e Meio Ambiente

Reviewed anonymously in the process of blind peer.
Received: 09/05/2020

Approved: 07/06/2020
Marcos Macri Olivera (iD)

Universidade Federal de Campina Grande, Brasil

http://lattes.cnpq.br/6161476125979644

http://orcid.org/0000-0001-9446-4727

macri.ccjs@gmail.com

Erivaldo Moreira Barbosa (iD)

Universidade Federal de Campina Grande, Brasil

http://lattes.cnpq.br/0405256524786499

http://orcid.org/0000-0001-7479-1827

erifat@terra.com.br
Referencing this:

OLIVEIRA, M. M.; BARBOSA, E. M.. Produção de pesquisa acadêmica internacional sobre gestão de recursos hídricos. Revista IberoAmericana de Ciências Ambientais, v.11, n.4, p.300-315, 2020. DOI: http://doi.org/10.6008/CBPC2179-6858.2020.004.0025 


\section{INTRODUÇÃO}

Em sua obra seminal Biological integrity: a long-neglected aspect of Water Resource Management, James R. Karr já alertava, devido ao incremento populacional e desenvolvimento tecnológico, que a água em qualidade e quantidade suficiente deveria ser uma problemática a receber esmerada atenção por parte da sociedade (KARR, 1991). Desde então, preocupações sobre escassez de água, inundações e o papel central do recurso hídrico na vida humana seguem obtendo destaque em estudos conceituados (BHATI et al., 2019; FANG et al., 2014; SANTANA et al., 2019). Soma-se a isso, a crescente escassez de água em escala global e o volume de efluentes de águas residuais de locais industriais e/ou aglomerações urbanas que estão se tornando fatores cada vez mais preocupantes em termos ambientais, econômicos e sociais (BAGATIN et al., 2014).

A água é considerada um recurso natural determinante na produção de alimentos de origem animal ou vegetal e não apenas um mero insumo. O seu grau de disponibilidade e distribuição dentro do sistema global pode facilitar ou inviabilizar a produção agropecuária, especialmente em regiões em que há ocorrência de secas ou distribuição anual irregular de chuvas, gerando, desta forma, perspectivas para o acentuamento da crise hídrica.

O uso eficiente da água com conhecimento adequado e a utilização de alternativas que otimizem sua utilização podem contribuir para aumentar a sua disponibilidade, reduzindo problemas de déficit provocados pelo aumento da demanda social em relação à oferta ambiental. Em linhas gerais, o acentuamento de variáveis como crescimento da população humana, mudanças climáticas, aumento do desperdício per capita e intensificação da concorrência nos mercados consumidores tornam imperativo que tecnologias avançadas sejam desenvolvidas e implementadas para a conservação e o gerenciamento otimizado dos recursos hídricos (BAGATIN et al., 2014; CARR et al., 2012).

Para enfrentar uma demanda crescente de água doce, estratégias diferentes se encontram em desenvolvimento. A primeira abordagem é destacada por Bagatin et al. (2014) e trata do uso aprimorado da água produzida como subproduto das atividades humanas relacionadas à agricultura. A segunda estratégia, destacam, volta-se para a recuperação e preservação das águas subterrâneas por tecnologias de limpeza ou por políticas e procedimentos preventivos. Finalmente, na última abordagem é apontada uma racionalização do problema da melhoria da eficiência da gestão da água em instalações industriais, com atenção especial aos recentes desenvolvimentos de novos algoritmos computacionais para otimização combinada de água/energia (BAGATIN et al., 2014).

Por outro caminho, Carr et al. (2012) apontam que a gestão de recursos hídricos ganhou impulso crescente nas últimas décadas, em especial a gestão participativa. Os autores mencionam documentos importantes que corroboram com essa temática, a exemplo da Declaração de Dublin sobre Água e Desenvolvimento Sustentável (adotada pela ONU em Janeiro de 1992) que incluiu a participação dos atores diretamente envolvidos no processo de gestão como um de seus princípios orientadores, além da Declaração do Rio (de Janeiro) sobre Meio Ambiente e Desenvolvimento e a Agenda 21, que também indicou o processo 
participativo como essencial para a gestão ambiental.

A necessidade de se pensar de forma interdisciplinar e participativa os aspectos ligados à gestão dos recursos hídricos, perpassam décadas e hoje, segundo Fernandes et al. (2008), esta problemática deve ser discutida e tratada através da compreensão da maneira pela qual a sociedade se relaciona com o meio ambiente, devendo ser compreendida como um problema econômico, social, cultural e espiritual, dependendo da corrente teórica e acadêmica como base para a escolha das melhores decisões pelo gestores e pela própria sociedade, que possui um papel determinante neste processo.

Apesar dos avanços em pesquisas e proposituras destacados sobre o tema, parece conveniente diagnosticar o campo de estudo, objetivando esclarecer marcos teóricos, assim como áreas de estudo, lacunas de pesquisa ou subcampos em desenvolvimento. Tal objetivo deve dar amparo conceitual para a construção de novos projetos de trabalho acerca da temática abordada. Posto isso, o presente estudo se justifica pela necessidade indicada. Para tanto, o uso de técnicas bibliométricas se posicionam como referenciais científicos no intento de conhecer e mapear os campos de conhecimento.

Nesse sentido, Koseoglu (2016) aponta que a bibliometria é o conjunto de métodos estatísticos utilizados para investigar a evolução da ciência e/ou disciplinas por meio da avaliação da performance de publicação de autores e instituições, bem como o mapeamento da estrutura e dinâmica dos campos de conhecimento por meio de dados extraídos de artigos, livros, proceedings, entre outros. Desta forma, nos estudos bibliométricos são utilizadas variáveis com o objetivo de capturar dados em suas fontes para receberem o devido tratamento estatístico, mapeando suas características e gerando indicadores de desempenho. Em alinhamento com a problemática emergente apresentada, o objetivo central deste trabalho é analisar a produção acadêmica internacional sobre a Gestão de Recursos Hídricos, utilizando como referência a base Web of Science.

\section{REVISÃO TEÓRICA}

Os ecossistemas lênticos são um recurso natural renovável inestimável para a biodiversidade, mas que podem ser alterados por atividades humanas e mudanças climáticas (GIARDINO et al., 2010). Em uma previsão severa, pode-se dizer que os efeitos potenciais das mudanças climáticas no suprimento e na qualidade da água afetarão todos os setores da economia, gerando impactos na saúde, agricultura, indústria, transporte, suprimento de energia, serviços ecossistêmicos não mercantis, pesca e florestas (OLMSTEAD, 2014).

Um dos caminhos apontados para solucionar essa problemática reside no desenvolvimento da gestão associada a esse recurso. Problemas complexos de gerenciamento podem ser resolvidos através de várias estratégias alternativas, como decisões, políticas ou ações, cada qual com diferentes características e consequências (ALAMANOS et al., 2018). Nesse contexto, uma gestão científica e eficaz dos recursos hídricos requer uma boa compreensão dos ciclos da água e uma integração sistemática de observações que possibilite melhores resultados de previsão (FANG et al., 2014).

Partindo desse ponto, Ajami et al. (2008) destacam que durante a década passada, houve um 
aumento no desenvolvimento de técnicas para minimizar as incertezas associadas aos recursos hídricos no mundo. Observa-se que o desenvolvimento de sistemas de suporte à decisão para aplicações ambientais é uma tarefa complexa e desafiadora e que a crescente complexidade dos problemas ambientais, o crescimento de atores envolvidos e o conflito de interesses tornam as decisões e o suporte à decisão dificultosos (MYSIAK et al., 2005).

Tal constatação pode impactar diretamente o resultado dos estudos de planejamento e gerenciamento de recursos hídricos, especialmente estudos de mudança climática que usam previsões climáticas (como precipitação e temperatura) para elaborar modelos hidrológicos e, consequentemente, projetar o impacto da mudança climática no gerenciamento de recursos hídricos (AJAMI et al., 2008; GEISSEN et al., 2015). Vale apontar que uma abordagem complementar se situa no planejamento de cenários - este baseado principalmente no processo de previsão de resultados possíveis no futuro (GUPTA et al., 2018).

Ziervogel et al. (2010) relatam que, embora o setor de água possa ter desafios específicos, o uso de previsões para melhorar a subsistência tem sido relevante em vários casos, inclusive no setor agrícola, tanto em escala de sobrevivência quanto no comercial, para gerenciar o nível de suprimentos. Apesar dos desafios no uso de informações climáticas, é necessário, para os mesmos autores, continuar explorando como as informações sobre a variabilidade climática podem ajudar a gerenciar os recursos hídricos, principalmente porque as respostas à variabilidade estão interligadas àquelas que lidam com o clima projetado. Corroborando, Yu et al. (2019) reforçam a importância de pesquisas que procuram desenvolver respostas para a variabilidade climática e que facilitam a projeção e implementação de ações voltadas para o controle da escassez e gerenciamento dos recursos disponíveis.

Aponta-se, em complemento, que as mudanças incluem variabilidade, provavelmente em uma escala sub-regional, mas não são detectáveis por modelos em escala global (ZIERVOGEL et al., 2010). Nesse sentido, encontrar respostas efetivas à variabilidade sazonal é fundamental e pode ser visto como uma estratégia para lidar com as mudanças climáticas de longo prazo (ZIERVOGEL et al., 2010). Ainda assim, é necessário destacar que apesar dos enormes esforços, o quadro que emerge sobre o status ecológico (em que se encontra o recurso hídrico) ainda é incompleto, fragmentado e com avaliações situacionais contraditórias (ALTENBURGER et al., 2015).

Para Carr et al. (2012) a participação do público na gestão de recursos hídricos é o fator chave para melhorar e restaurar a qualidade dos recursos hídricos degradados. O gerenciamento integrado de recursos hídricos (GIRH) é fortemente promovido no setor de água, tendo em vista sua intenção em desenvolver soluções holísticas. O elemento chave é a participação do público, que é altamente importante nas áreas urbanas devido à densidade populacional. Quaisquer mudanças no padrão urbano afetam a população em maior escala (CARR et al., 2012). Em uma escala local, por exemplo, a Política Nacional de Recursos hídricos (BRASIL, 1997) aponta para a necessidade de participação na gestão ao mencionar, em suas diretrizes gerais de ação, a obrigatoriedade de articulação do planejamento de recursos hídricos com os setores usuários.

É possível concluir que, com a crescente crise hídrica no mundo, uma abordagem integrada que leve em consideração todos os parâmetros físicos, biológicos, químicos e sociais na gestão de recursos hídricos é 
essencial para promover a sustentabilidade dos recursos (CARR et al., 2012; MASHAZI et al., 2019). Tal conceito de integração na gestão de recursos hídricos já tem obtido incorporação na legislação associada à água de alguns países, como no caso da África do Sul (ESTERHUIZEN et al., 2018).

\section{MATERIAIS E MÉTODOS}

Considerando que objetivou proporcionar uma visão geral sobre o elemento em foco no estudo (do tipo aproximativo) e que, ainda assim, procurou descrever as características deste mesmo objeto, esta pesquisa se caracteriza como exploratória e descritiva (GIL, 2008). Também possui uma natureza bibliométrica, tendo em vista que buscou investigar a evolução do tema em foco, por meio da avaliação de performance de publicação de autores, além de mapear a estrutura do campo de conhecimento por meio de dados (KOSEOGLU, 2016). Para tanto, fez-se uso de técnicas avaliativas (medidas de produtividade e métricas de impacto) e técnicas relacionais (coautoria e coocorrência).

Com relação ao procedimento da pesquisa, foi desenvolvido em etapas. Inicialmente, escolheu-se o tema central da pesquisa: Gestão de Recursos Hídricos. Tal escolha se deu em função deste estar alinhado centralmente com o tema do Projeto de Tese de Doutorado do autor principal deste trabalho (realizado no Programa de Pós-Graduação em Engenharia e Gestão de Recursos Naturais da Universidade Federal de Campina Grande - UFCG). Dessa maneira, o estudo aqui realizado se constituiu em uma etapa preliminar de pesquisa e construção do referencial teórico do referido projeto.

Decidido o tema, realizou-se a pesquisa inicial das publicações. Utilizou-se como base a Web of Science (v. 5.34). A pesquisa foi realizada em 25 de março de 2020. Foram escolhidos os seguintes índices de pesquisa (coleções): Science Citation Index Expanded (SCI-EXPANDED), Social Sciences Citation Index (SSCI) e Emerging Sources Citation Index (ESCI). Estes foram escolhidos em função do alinhamento de suas bases com o tema escolhido para o estudo. Foram selecionados para a pesquisa todos os anos disponíveis nas bases (1945 a 2020) como maneira de dar oportunidade ao máximo possível de publicações. Pesquisou-se publicações que contivessem 'Gestão de Recursos Hídricos' (Water Resource Management) em seus títulos - essa escolha procurou dar mais solidez ao universo de dados recolhidos, evitando publicações que tivessem a referida expressão em palavras-chaves ou resumos, mas não fosse elementos representativos no texto da publicação. Como resultado desse procedimento foram identificados 416 estudos. Em seguida foram realizados refinamentos, buscando aprimorar a investigação: mantiveram-se apenas as publicações do tipo article e review e as com idioma inglês. Dessa maneira, o total de publicações selecionados foram 358 . 0 script final da busca foi: 'Web of Science Core Collection. Você pesquisou por: TíTULO: ('water resource management') Refinado por: [excluindo] tipos de documento: (early access) and tipos de documento: (article or review) and idiomas: (english). Tempo estipulado: Todos os anos. Índices: SCI-EXPANDED, SSCI, ESCI'.

O tratamento dos dados foi a terceira etapa do método. Quanto às técnicas avaliativas, os dados foram tabulados e organizados utilizando a ferramenta Clarivate Analytics (disponível na Web of Science). Foram gerados gráficos descrevendo publicações por áreas, por ano, por periódicos, por organizações, por países e por idiomas. As publicações também foram ranqueadas a partir do 'cit. Score' e classificadas de 
acordo com seu Fator $\mathrm{H}$ (H-Index) para identificar quais autores e, por consequência, obras possuem impacto na área de estudo. Hirsch (2005) propõe que um pesquisador possui Fator $\mathrm{H}$ se seu Np (número de publicações) tiver um mínimo de $h$ citações, cada um deles, e, os demais estudos, tiverem menos ou iguais citações cada um. Faz-se interessante mencionar que o Fator $\mathrm{H}$ foi utilizado em dois momentos: no primeiro se constatou o $\mathrm{H}$-index levando em consideração as citações dentro e fora do grupo de publicações identificado no procedimento de coleta de dados e, em um segundo momento, verificou-se o $\mathrm{H}$-index considerando as citações apenas dentro do grupo. Quanto às técnicas relacionais, utilizou-se o software VOSviewer version 1.6.12. O referido programa é um software que oferece ferramentas para construção e visualização de redes bibliométricas (VAN ECK et al., 2017) e, a partir deste, foram formulados mapas bibliométricos com base nos tipos e unidades de análise listados no quadro 1.

Quadro 1: Definição dos parâmetros de corte no VOSviewer

\begin{tabular}{|l|l|l|}
\hline \multicolumn{2}{|l|}{ PARÂMETROS } & DESCRIÇÃO \\
\cline { 1 - 2 } $\begin{array}{l}\text { Tipo de } \\
\text { Análise }\end{array}$ & $\begin{array}{l}\text { Unidade } \\
\text { Análise }\end{array}$ & $\begin{array}{l}\text { Relação de força que ocorre quando, em síntese, autores participam de publicações em } \\
\text { parceria. }\end{array}$ \\
\hline Co-authorship & Authors & $\begin{array}{l}\text { Relação de força que ocorre quando, em síntese, organizações participam de publicações } \\
\text { em parceria. }\end{array}$ \\
\hline Co-authorship & Organizations & $\begin{array}{l}\text { Relação de força que ocorre quando, em síntese, países participam de publicações em } \\
\text { parceria. }\end{array}$ \\
\hline Co-authorship & Countries & Relação de força e frequência entre palavras-chave (termos). \\
\hline Co-occurrence & All Keywords &
\end{tabular}

Fonte: Adaptado de Van Eck et al. (2017).

Cabe destacar que os mapas gerados enfatizam a formação de clusters. As técnicas de clusterização se apresentam como instrumentos proeminentes no campo da pesquisa bibliométrica, sendo utilizados para identificar grupos de relacionamento entre publicações, autores ou fontes de publicação (VAN ECK et al., 2017).

A última etapa do método de pesquisa consistiu em uma análise qualitativa a partir das técnicas avaliativas e relacionais aplicadas na etapa anterior. Foram observados os dados mais destacados e procurouse evidenciar aspectos relevantes, assim como concluir sobre as relações de colaboração e clusterização dos termos componentes mais alinhados no campo em estudo.

\section{RESULTADOS E DISCUSSÃO}

De maneira geral, das 358 publicações localizadas, a mais antiga é Water resource management Economic, conservation, and legal views, escrito por J. A. Crutchfield, J. A. Biggs e R. W. Johnson e publicado no Journal Water Pollution Control Federation, em 1964, e a mais recente é Achieve Sustainable development of rivers with water resource management - economic model of river chief system in China, cuja autoria pertence à Xiaojing Liu e mais 4 autores e publicado no Science Of The Total Environment, em março do corrente ano (2020). A média de citações por publicação é de 19 com um total de 6.861 citações. Um panorama detalhado da evolução de publicações por ano é apresentado na figura 1. 


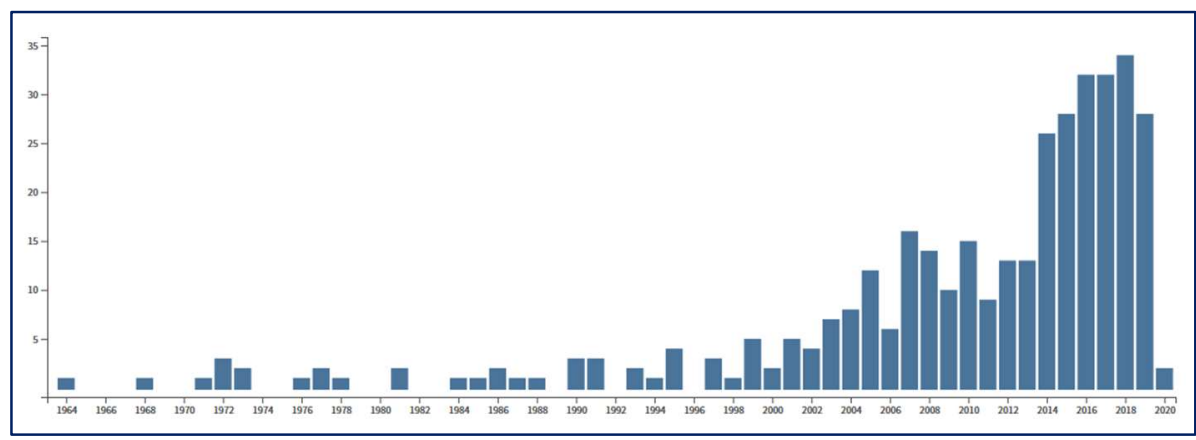

Figura 1: Publicações por ano.

Conforme é possível observar, o nível de publicações se manteve em um patamar inicial (limite de 5 por ano) desde seu começo até início dos anos 2000. Em 2003, ocorre um ponto de inflexão com a publicação de 7 trabalhos. A partir daí o nível de publicações passa a apresentar uma clara tendência de aumento. Em 2014, a quantidade de publicações atinge seu patamar atual, saltando para 26 publicações e alcançando 34 em 2019. Obviamente, os dados apresentados na figura indicam um crescente aumento de interesse em pesquisa pelo tema, com especial representatividade para a década 2010. Parece adequado, ainda, interpretar que isso seja reflexo da popularização de discussões sobre as questões ambientais, entre outros aspectos. Nesse ponto, cabe analisar quais áreas de pesquisa têm dirimido atenção ao tema. Os dados relativos a isso se encontram na figura 2 .

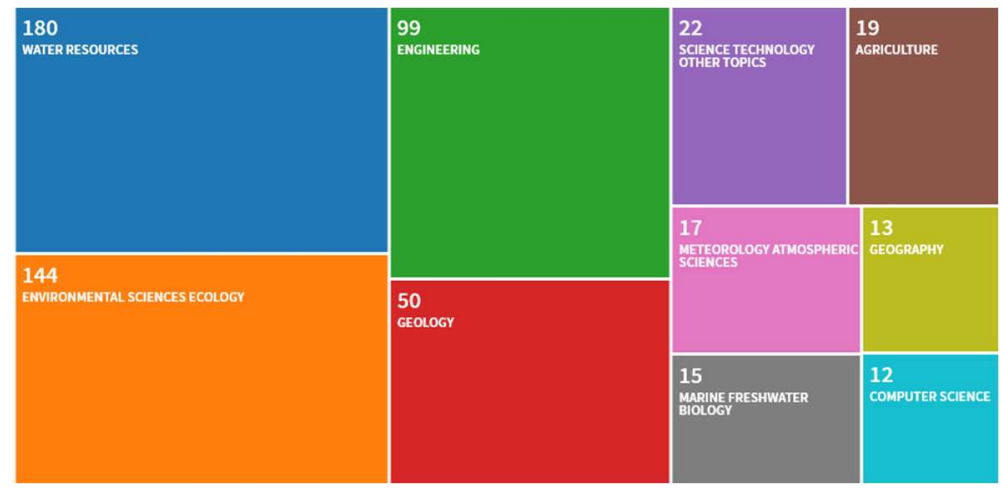

Figura 2: Publicações por áreas.

A figura 2 apresenta as 10 áreas que detêm mais publicações sobre Gestão de Recursos Hídricos. Vale informar que uma mesma publicação pode estar classificada em mais de uma área. Dessa forma, mais de 50\% das publicações identificadas são fruto de pesquisa na área de Water Resources e mais de $40 \%$ em Environmental Sciences Ecology, ou seja, mais de 90\% das publicações se encontram classificadas em, ao menos, uma dessas áreas. Engenharia e Geologia também são áreas de destaque nas publicações. Também estão presentes áreas tradicionais (à exemplo da Agricultura), assim como áreas de surgimento mais recente, como Imaging Science Photographic Technology. Essa pluralidade latente das áreas que se interessam pelo tema corrobora com o aspecto interdisciplinar desse campo. Nesse caminho, o elemento plural também se manifesta ao se verificar quais organizações são responsáveis por publicações em Gestão de Recursos Hídricos - o que pode ser visualizado na figura 3. 


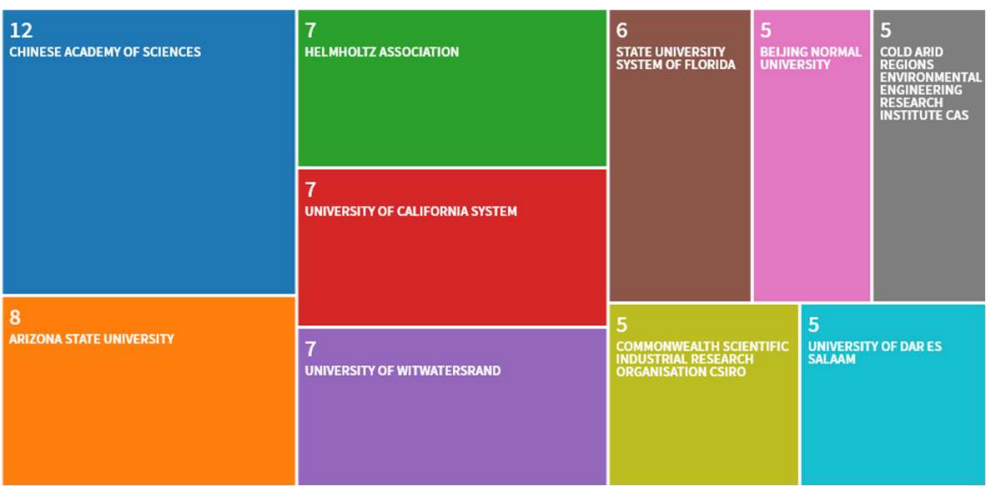

Figura 3: Publicações por organizações.

Embora com um percentual ainda pequeno em relação à totalidade de publicações (pouco mais de $3 \%)$, a organização com mais destaque entre as 10 que mais publicaram sobre a temática em estudo foi a Chinese Academy Sciences. China e EUA possuem destaque nesse grupo, totalizando mais da metade de organizações pesquisadoras. Apesar da intensa fragmentação observada, dois aspectos merecem destaque na análise: a ausência de organizações europeias entre o grupo de 10 com mais publicações e a ausência de organizações brasileiras no grupo total das que publicam, principalmente em se considerando que o Brasil possui $12 \%$ das reservas de água doce superficial do mundo e alguns dos maiores reservatórios subterrâneos de água líquida (ANA, 2015). Esse ponto nos remete a analisar a nacionalidade das pesquisas abordadas neste estudo. Na figura 4, pode-se verificar o grupo de 10 países com mais publicações no universo de trabalhos pesquisado.

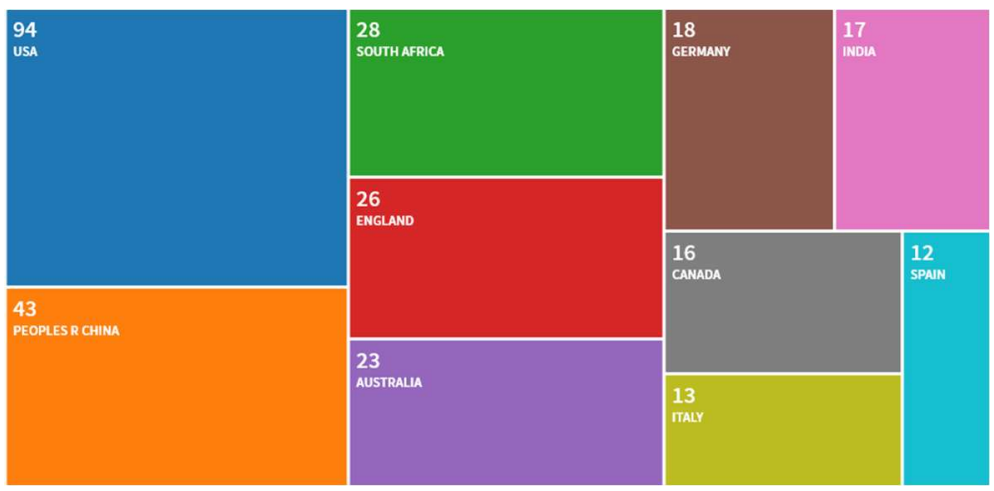

Figura 4: Publicações por países.

Conforme se observa, os EUA é o país com mais publicações (mais de $26 \%$ do total). Em consonância com os dados anteriores, a China aparece em segundo, com $12 \%$ das publicações. De forma geral, entre os 10 primeiros, 4 são europeus e nenhum das Américas Central e do Sul. O Brasil se localiza em um grupo secundário, com 11 publicações, mais que Holanda, Suécia, Japão e outros. Até o ponto presente, os dados, de forma ampla, reforçam o papel de protagonismo para EUA e China no cenário da pesquisa sobre Gestão de Recursos Hídricos. Concluindo o elemento avaliativo da investigação, a figura 5 apresenta o grupo de 5 revistas com mais publicações sobre a temática aqui abordada.

Nota-se grande equilíbrio na quantidade de publicações dos principais periódicos que recebem publicações sobre Gestão de Recursos Hídricos. As revistas Water e Water SA detêm quase 8\% do total de pesquisas publicadas. São sediadas, respectivamente, na Suíça e África do Sul. Duas das cinco são holandesas 
(Water Resources Management e Journal of Hydrology) e a restante americana. Ao todo, aproximadamente, 170 revistas publicaram pesquisas sobre o tema em estudo. Para avaliar com mais propriedade as principais referências, em termos de publicações, utilizou-se o Fator $\mathrm{H}$ ( $H$-index) para concluir sobre as obras de maior impacto no campo delimitado pelos temas-chave abordados neste estudo. Esta foi feita em duas etapas: na primeira considerou-se o Fator $\mathrm{H}$ de cada publicação tomando por base as citações realizadas por publicações que compõem e que não compõem o universo dos 358 trabalhos elencados na base Web of Science; e na segunda tomou-se por base as citações realizadas apenas por publicações que compõem o universo. Dessa maneira, os resultados são apresentados nos quadros 2 e 3.

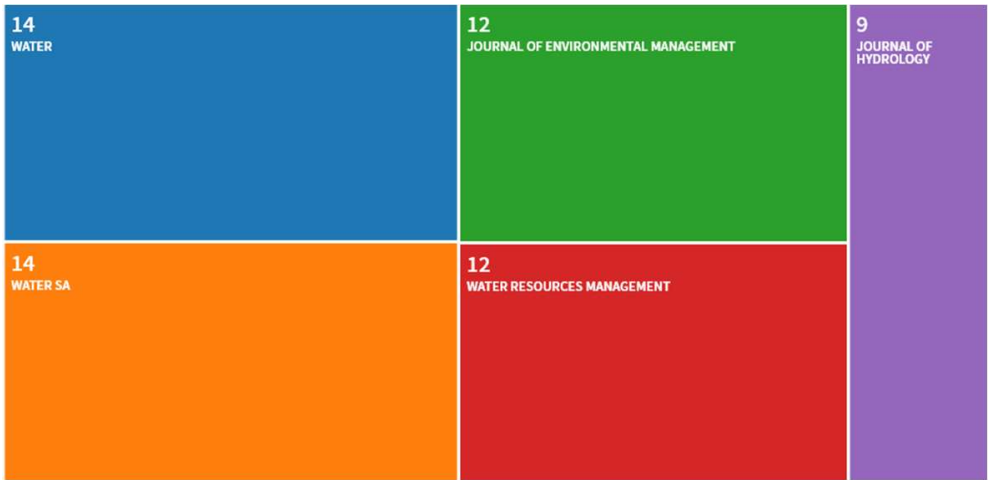

Figura 5: Publicações por periódicos.

Quadro 2: Publicações de maior impacto (H-index).

\begin{tabular}{|l|l|l|l|l|}
\hline Título & $\begin{array}{l}\text { Primeiro } \\
\text { Autor }\end{array}$ & Título da Fonte & Ano & Citações \\
\hline $\begin{array}{l}\text { Biological integrity - a long-neglected aspect of water-resource } \\
\text { management }\end{array}$ & Karr, J. R. & Ecological Applications & 1991 & 820 \\
\hline $\begin{array}{l}\text { Historical temporal trends of hydro-climatic variables and } \\
\text { runoff response to climate variability and their relevance in } \\
\text { water resource management in the Hanjiang basin }\end{array}$ & Journal Of Hydrology & 2007 & 187 \\
\hline $\begin{array}{l}\text { Comparison of multiple criteria analysis techniques for water } \\
\text { resource management }\end{array}$ & $\begin{array}{l}\text { Hajkowicz, } \\
\text { Stefan }\end{array}$ & $\begin{array}{l}\text { European Journal Of } \\
\text { Operational Research }\end{array}$ & 2008 & 185 \\
\hline $\begin{array}{l}\text { Emerging pollutants in the environment: A challenge for water } \\
\text { resource management }\end{array}$ & $\begin{array}{l}\text { Geissen, } \\
\text { Violette } \\
\text { Water Conservation } \\
\text { Research }\end{array}$ & 2015 & 184 \\
\hline $\begin{array}{l}\text { Bayesian Networks and participatory modelling in water } \\
\text { resource management }\end{array}$ & Castelletti, A. & $\begin{array}{l}\text { Environmental Modelling } \\
\text { \& Software }\end{array}$ & 2007 & 171 \\
\hline $\begin{array}{l}\text { Towards the development of a decision support system for } \\
\text { water resource management }\end{array}$ & Mysiak, J. & $\begin{array}{l}\text { Environmental Modelling } \\
\text { \& Software }\end{array}$ & 2005 & 165 \\
\hline $\begin{array}{l}\text { Integrated water resource management, institutional } \\
\text { arrangements, and land-use planning }\end{array}$ & Mitchell, B. & $\begin{array}{l}\text { Environment And Planning } \\
\text { A }\end{array}$ & 2005 & 158 \\
\hline $\begin{array}{l}\text { Regional applications of an index of biotic integrity for use in } \\
\text { water-resource management }\end{array}$ & Miller, D.L. & Fisheries & 1988 & 146 \\
\hline $\begin{array}{l}\text { Future water quality monitoring - Adapting tools to deal with } \\
\text { mixtures of pollutants in water resource management }\end{array}$ & $\begin{array}{l}\text { Altenburger, } \\
\text { Rolf }\end{array}$ & $\begin{array}{l}\text { Science Of The Total } \\
\text { Environment }\end{array}$ & 2015 & 139 \\
\hline $\begin{array}{l}\text { A review of the methods available for estimating soil moisture } \\
\text { and its implications for water resource management }\end{array}$ & $\begin{array}{l}\text { Dobriyal, } \\
\text { Pariva }\end{array}$ & \begin{tabular}{l} 
Journal Of Hydrology \\
\hline
\end{tabular} & 2012 & 126 \\
\hline
\end{tabular}

A temática estudada, delimitada de acordo com os parâmetros estabelecidos nos procedimentos metodológicos, possui um $\mathrm{H}$-index igual a 41. Isso indica que 41 publicações são consideradas trabalhos de impacto no campo estudado. No quadro 2 se encontram listadas as 10 principais obras, ou seja, as 10 com maior H-index. A obra considerada de maior impacto é Biological integrity - a long-neglected aspect of waterresource management, escrita por J. R Karr e publicada na revista Ecological Applications, em 1991. A disparidade de citações entre essa obra e a seguinte (820 contra 187), no ranqueamento de fator $\mathrm{H}$, indica 
uma relevância de imenso destaque para a mesma. Esse mesmo autor, ainda possui uma segunda publicação ranqueada entre as 10 primeiras (Regional applications of an index of biotic integrity for use in water-resource management). São estas duas justamente apenas as duas publicações de impacto (entre as 10 mais) que foram publicadas no século passado. Nenhuma publicação brasileira consta nesse patamar. Em seguida, pode-se analisar a segunda composição de $H$-index, apenas com citações realizadas por publicações que constam no universo de trabalhos investigado.

Quadro 3: Publicações de maior impacto (H-index).

\begin{tabular}{|l|l|l|l|l|}
\hline Título & $\begin{array}{l}\text { Primeiro } \\
\text { Autor }\end{array}$ & Título da Fonte & Ano & Citações \\
\hline $\begin{array}{l}\text { Biological integrity - a long-neglected aspect of water- } \\
\text { resource management }\end{array}$ & Karr, J.R. & Ecological Applications & 1991 & 3 \\
\hline $\begin{array}{l}\text { Integrated water resource management, institutional } \\
\text { arrangements, and land-use planning }\end{array}$ & Mitchell, B. & Environment And Planning A & 2005 & 3 \\
\hline $\begin{array}{l}\text { Bayesian Networks and participatory modelling in water } \\
\text { resource management }\end{array}$ & Castelletti, A. & $\begin{array}{l}\text { Environmental Modelling \& } \\
\text { Software }\end{array}$ & 2007 & 3 \\
\hline
\end{tabular}

O novo quadro corrobora com a análise anterior. A principal obra permanece sendo a de J. R. Karr. Os trabalhos Integrated water resource management, institutional arrangements, and land-use planning e Bayesian Networks and participatory modelling in water resource management complementam o grupo de obras de impacto - agora reduzido para apenas 3 publicações. Uma análise qualitativa detalhada seria indicada para investigar por quais razões as obras do campo são pouco citadas pelas próprias obras que compõem o campo. Indícios da natureza dessa relação dentro do campo podem ser visualizados através da composição relacional de autores, organizações e países que produzem pesquisa na área objeto deste estudo. Dessa maneira, utilizou-se como ferramenta de visualização de redes bibliométricas o software VOSviewer version 1.6.12. Com o auxílio deste, foram elaborados mapas de acordo com os parâmetros de corte informados no quadro 1.

Para fins de análise, deve-se observar que o tamanho da esfera de cada autor é determinado pelo seu peso em termo de citações, de maneira que quanto mais citações o autor recebeu, maior será sua esfera. A proximidade entre esferas de autores representa sua proximidade em termos de semântica - uma análise aprofundada sobre o conteúdo das obras desses autores, em casos assim, pode concluir se essa proximidade semântica representa afinidade intelectual. As conexões entre as esferas representam trabalho em cooperação, expressas em coautoria de obras. E, por fim, um conglomerado de esferas com a mesma cor representa um conjunto de autores interligados entre si por relações de coautoria, aqui abordados pelo software como clusters.

A figura 6, apresenta a rede bibliométrica de coautoria, por autores, no universo de publicações investigado. A rede completa possui um total de 1.200 autores. A formatação da rede foi limitada à visualização dos 500 autores mais representativos da mesma, como forma de otimizar a configuração visual da mesma e permitir uma análise mais aprofundada. 


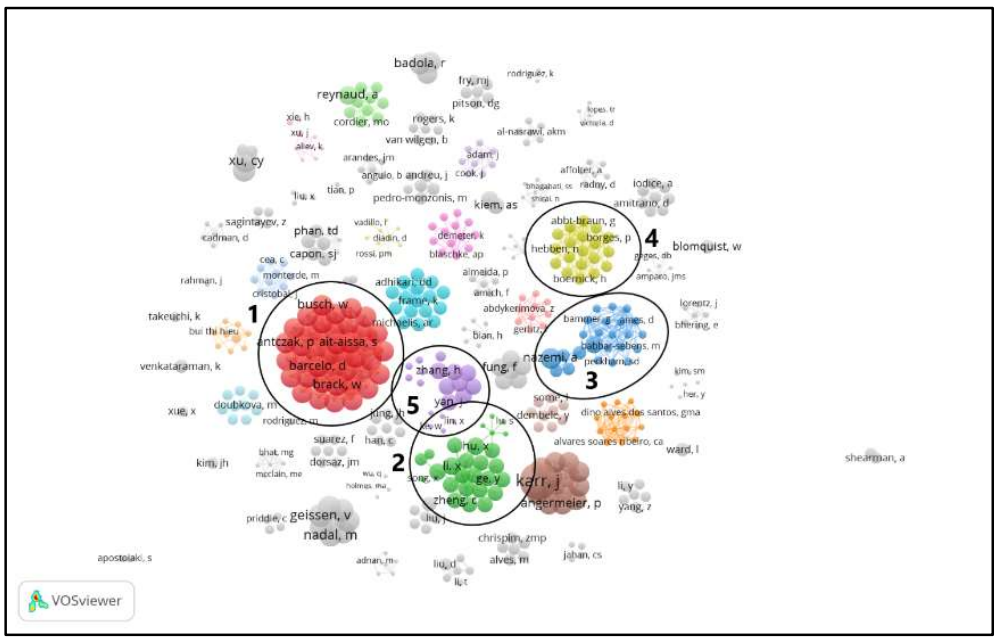

Figura 6: Mapa bibliométrico de coautoria, por autores.

A rede bibliométrica de coautoria, por autores, do campo em estudo apresenta um total de 66 clusters de cooperação. Os 5 principais estão destacados com círculo. O principal deles, em vermelho, é composto por 41 autores. Trata-se de um conglomerado incomum de cooperação, visto que não se trata de um grupo de autores que realizam pesquisas em múltiplas parcerias dentro do cluster e sim de uma única publicação com 41 autores. Trata-se da publicação Future water quality monitoring - Adapting tools to deal with mixtures of pollutants in water resource management, já mencionada como uma das que possuem maior fator de impacto entre as pesquisadas (quadro 2). O segundo cluster (em verde na rede) é composto por 27 autores. Possui, em sua maioria, autores chineses, sendo o de maior destaque (em termos de menções) X. Hun (80 citações). O terceiro, quarto e quinto clusters possuem, respectivamente, 24, 19 e 19 autores. É possível observar que J. R. Karr, apesar de ser o autor mais citado na rede inteira, participa de um cluster de impacto médio na rede. Também cabe salientar que a análise visual permite confirmar a pouca integração entre os clusters da rede, indicando blocos de pesquisa que pouco se comunicam. Nesse sentido, a figura 7, apresenta um overlay visualization, caracterizando a composição dos clusters de acordo com sua cronologia de constituição.

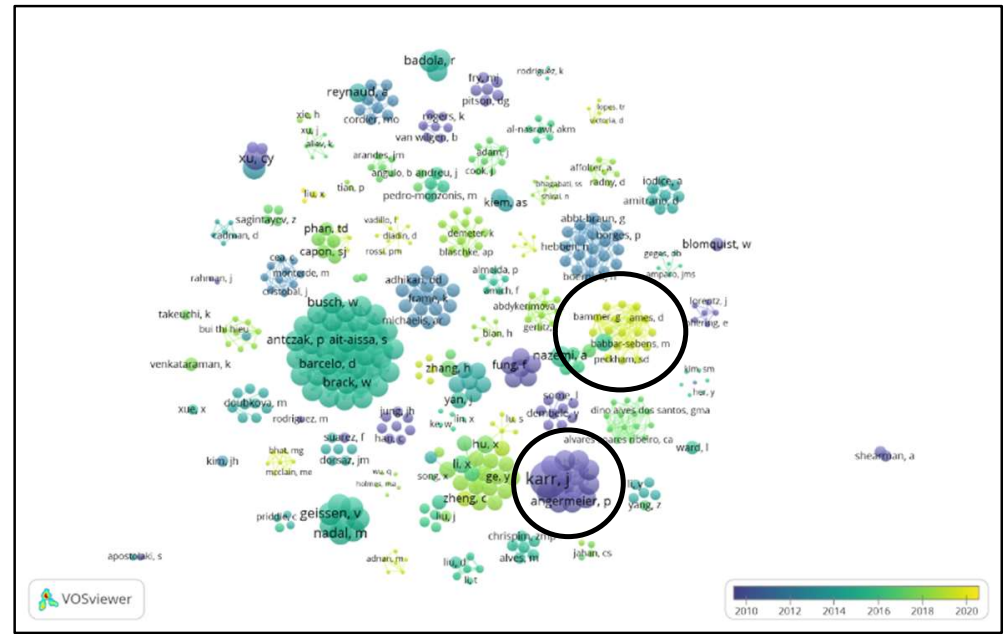

Figura 7: Mapa bibliométrico de coautoria, por autores (overlay visualization)

Observa-se que, dos principais conglomerados destacados na figura 6, o que apresenta publicações mais recentes é o terceiro e o menos atual é o que tem como componente J. R. Karr. Essas observações 
podem indicar novas tendências ou subcampos de conhecimento se formando, dentro do campo geral, e que estão em desenvolvimento, assim como temáticas que podem ter encontrado seu esgotamento ou, por motivos quaisquer, deixaram de despertar interesse dos autores componentes dos conglomerados. Nesse ponto, verificam-se formações de autores com uma configuração de publicações atual, o que corrobora com a afirmação inicial do aumento crescente de interesse por pesquisa no tema. Um ponto de vista sequencial é apresentado na figura 8, em que é apresentada a rede de coautoria por organizações.

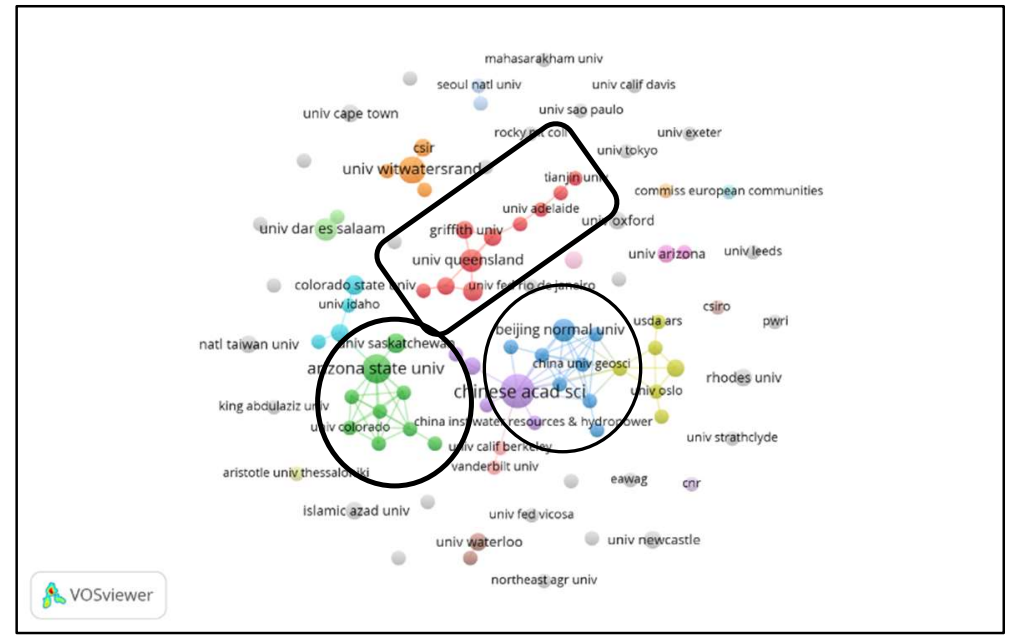

Figura 8: Mapa bibliométrico de coautoria, por organizações.

Aqui é possível fazer observações sobre os principais grupos de organizações que pesquisam em parceria sobre a Gestão de Recursos Hídricos. Exatamente, estão compostos 49 clusters de cooperação na rede. Os 3 principais se encontram circulados. O cluster em vermelho é o que possui mais componentes (10). A Universidade de Queensland, na Austrália, é a mais destacada em termos de publicações e mantém parcerias sólidas com a Griffith University, Monash University e outras também australianas. O segundo cluster (em verde na figura) é composto por 9 organizações e tem como elemento de destaque em publicações a Arizona State University, do estado do Arizona (EUA). Este, por sua vez, se configura como um conglomerado essencialmente norte-americano de pesquisa. Por último, em destaque no mapa em azul, é possível constatar um conglomerado de organizações mais 'internacionalizado', com organizações chinesas com significativa aparição (Beijing Normal University e China University of Geosciences), da Rússia (Russian Academy of Sciences), Europa e EUA - certamente um dos grupos com mais globalizados da rede. Apesar de tal constatação, a maior parte dos conglomerados é pequena e com pouca proximidade com outros grupos. Essa problemática abre espaço para analisar a cooperação entre as nações. Dessa maneira, a figura 9, apresenta a rede bibliométrica de coautoria por países.

A rede por países tem menos clusters e apresenta uma maior integração que a de organizações. 0 principal conglomerado (em vermelho) possui 8 países e tem como destaque os EUA, tendo como parceiros próximos a Itália, Hungria e Gana. A Grécia também se destaca nesse agrupamento. O segundo conglomerado mais significativo é mencionado em verde na rede. Suécia e Brasil lideram o grupo em termos de publicações. Apenas Brasil e Singapura não são europeus nesse cluster. A partir daí os grupos ficam menores. Nestes, observa-se com destaque a China, Alemanha, Austrália e África do Sul. Alguns países, a 
exemplo da Turquia e Jamaica, se encontram em regiões periféricas na rede, denotando carência de aprofundamento no estudo da temática.

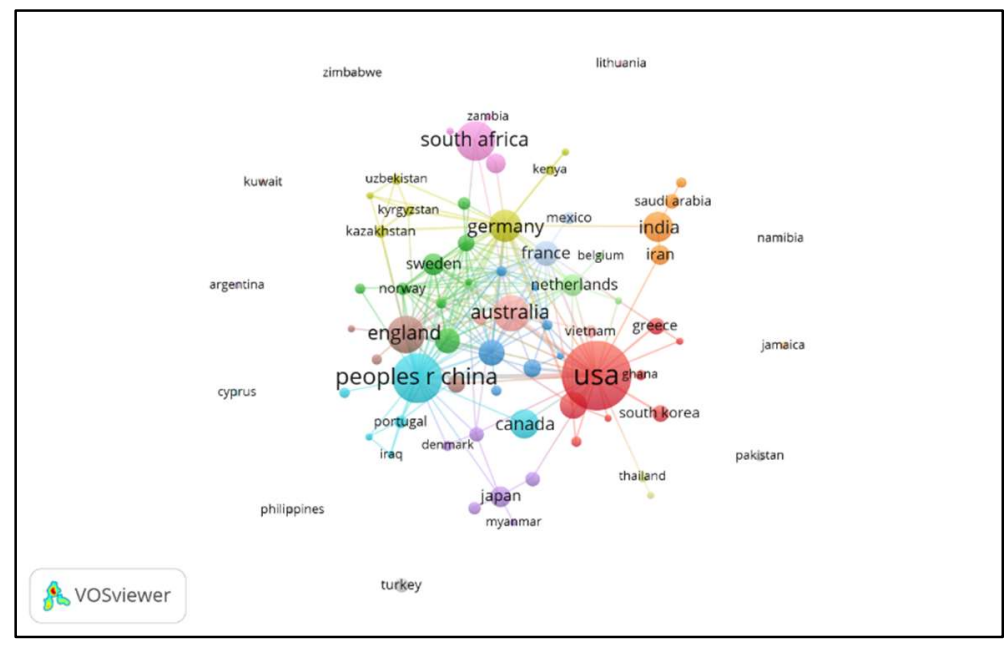

Figura 9: Mapa bibliométrico de coautoria, por países.

Complementando a análise relacional, cabe estudar de que maneira a temática tem formado agrupamentos de subtemas, ou seja, quais termos e assuntos são tratados em conjunto delineamento arcabouços que permitem compreender a lógica narrativa utilizada pelos autores do campo em estudo. Tal procedimento pode ser realizado utilizando os mapas bibliométricos de coocorrência. Nesse sentido, a figura 9 apresenta o mapa de coocorrência de termos para o tema Gestão de Recursos Hídricos.

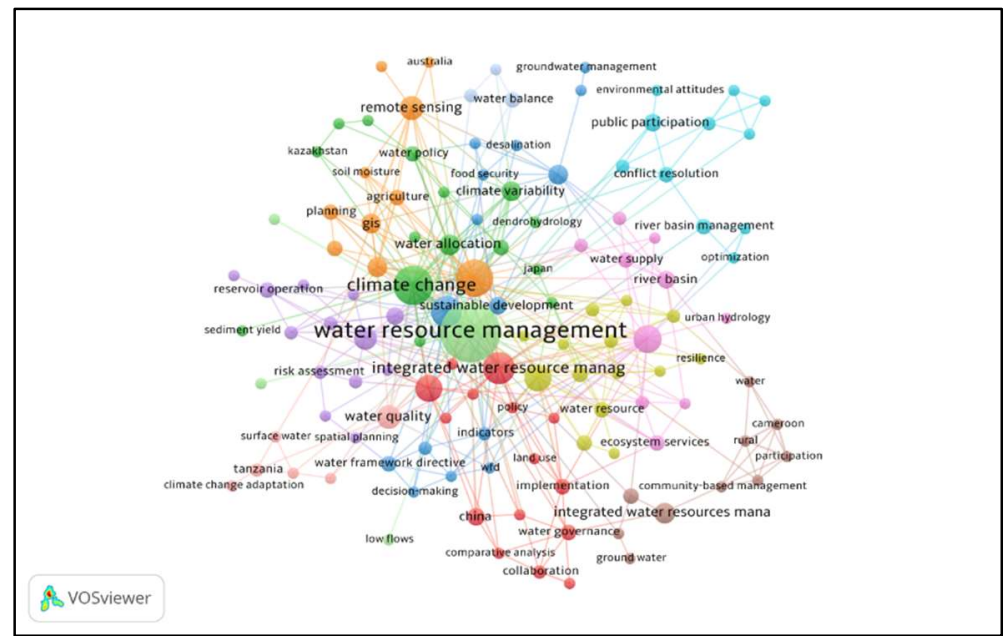

Figura 10: Mapa bibliométrico de coocorrência, por termos.

O mapa de coocorrência foi elaborado considerando apenas os termos que constam nas palavraschave dos trabalhos investigados. Dessa maneira, foram identificados 126 termos que foram mencionados mais de uma vez (os termos com apenas umas menções foram desconsideradas). Tal escolha se justifica para dar mais solidez à formatação do mapa, evitando uma formatação excessivamente fragmentada (em virtude do volume extenso e variado de termos mencionados nos abstracts das publicações). Conforme se pode observar, por motivos lógicos, a Gestão de Recursos Hídricos se encontra no centro da rede e conectada a todos os clusters. Cabe lembrar que a conexão entre dois termos simboliza sua utilização conjunta em publicações e quanto mais proximidade entre os termos, maior alinhamento semântico - isso pode 
caracterizar, por exemplo, uma afinidade intelectual entre as publicações. Quanto aos agrupamentos em si, percebe-se a formação de 12 conglomerados de termos. O cluster em vermelho no mapa é composto por 15 termos e tem como tema central a 'Gestão Integrada de Recursos Hídricos'. Assuntos como 'gestão da água', 'políticas', 'colaboração', 'pressão da população', 'governança da água' e outros relacionados estão presentes no cluster. Nota-se um subtema voltado para discussão de elementos que impactam a integração da gestão dos recursos hídricos e o papel do Estado nesse gerenciamento. O segundo conglomerado em destaque (em vede no mapa) também possui 15 termos e é elaborado tendo como termo-chave as mudanças climáticas. A mudança de clima, irrigação na agricultura, a distribuição da água, a seca, fluxo de água e cooperação são temas recorrentes nesse aglomerado. Conclui-se por um subtema que discute a mudança climática e como esta impacta a gestão de recursos hídricos, elencando raízes e efeitos. O terceiro cluster de destaque (em azul no mapa) também possui 15 itens e tem como elemento mais destacado a gestão do recurso hídrico. Estão associados os termos 'sistema de suporte às decisões', 'tomada de decisão', 'segurança energética', 'segurança alimentar', 'segurança hídrica', 'lençóis freáticos', 'administração dos lençóis freáticos', 'desenvolvimento sustentável' e 'indicadores'. Aqui tem um agrupamento discutindo o tema mencionado com foco no processo de tomada de decisão sobre os recursos hídricos, além da discussão sobre pontos de convergência desejáveis (a exemplo da segurança nos aspectos alimentares, energéticos e hídricos). Um outro agrupamento de destaque que caminhou em outra direção da temática está destacado em roxo no mapa e tem como elemento central o termo 'incerteza'. Estão associados a esta os termos 'stakeholders', 'monitoramento', 'cenários', 'operação de reservatórios', 'modelagem participativa', 'otimização de múltiplos objetivos' e outros. Neste grupo é possível notar uma discussão sobre o processo de tomada de decisão considerando a incerteza do processo decisório e abordando a utilização de técnicas ou ferramentas lógico-matemáticas para auxiliar no processo de planejamento.

Como encerramento, é interessante frisar que uma análise com mais detalhamento de todos os clusters resultará em um mapeamento mais completo e aprofundado do campo conceitual de Gestão de Recursos Hídricos. Conforme se observa na figura 9, de forma geral e apesar da classificação por clusters, o mapa bibliométrico apresenta intensa proximidade e, em alguns pontos, até sobreposição dos mesmos, o que indica uma integração dos subtemas desenvolvidos no campo, por ora.

\section{CONCLUSÕES}

O objetivo deste artigo foi analisar a produção de pesquisa internacional sobre Gestão de Recursos Hídricos - tomando como referência as publicações que compõem a base Web of Science. Para tanto, realizou-se um estudo bibliométrico em que se lançou mão de técnicas avaliativas e relacionais. Dessa forma, 358 publicações foram selecionadas e analisadas.

Em termos avaliativos, os resultados apontam para uma tendência crescente da produção internacional sobre o tema, com clara acentuação na década 2010. EUA e China são os países com mais autores pesquisadores. Há uma significativa fragmentação de organizações que promovem pesquisas, o mesmo ocorrendo com as revistas que publicam. $\mathrm{O}$ fator $\mathrm{H}$ ( $\mathrm{H}$-index) aponta para 41 publicações que podem 
ser consideradas referência na área, sendo a de maior impacto Biological integrity - a long-neglected aspect of water-resource management, escrita por J. R Karr e publicada na revista Ecological Applications, em 1991. Por outro lado, em termos relacionais, a análise se fundamentou na construção e avaliação de mapas bibliométricos com a utilização do software VOSVIEWER. Os resultados sobre coautoria (entre autores, organizações e países) e coocorrência indicam uma produção diversificada com clara clusterização. A estrutura semântica do campo sugere a formação de subtemas integrados e que discutem os aspectos da Gestão de Recursos Hídricos de forma ampla.

\section{REFERÊNCIAS}

ANA. Agência Nacional de Águas. Disponibilidade de demanda de Recursos Hídricos no Brasil: estudo técnico. Caderno de Recursos Hídricos. Brasília: ANA, 2015.

AJAMI, N. K.; HORNBERGER, G. M.; SUNDING, D. L.. Sustainable water resource management under hydrological uncertainty. Water Resources Research, v.44, n.11, 2008. DOI: https://doi.org/10.1029/2007wr006736

ALAMANOS, A.; MYLOPOULOS, N.; LOUKAS, A.; GAITANAROS, D.. An Integrated Multicriteria Analysis Tool for Evaluating Water Resource Management Strategies. Water, v.10, n.12, 2018. DOI: https://doi.org/10.3390/w10121795

ALTENBURGER, R.; AIT-AISSA, S.; ANTCZAK, P.; BACKHAUS, T.; BARCELÓ, D.; SEILER, T.-B.; BRACK, W.. Future water quality monitoring: Adapting tools to deal with mixtures of pollutants in water resource management. Science of The Total Environment, v.512-513, p.540-551, 2015. DOI: https://doi.org/10.1016/j.scitotenv.2014.12.057

BAGATIN, R.; KLEMEŠ, J. J.; REVERBERI, A. P.; HUISINGH, D.. Conservation and improvements in water resource management: a global challenge. Journal of Cleaner Production, v.77, p.1-9, 2014. DOI:

https://doi.org/10.1016/j.jclepro.2014.04.027

BHATTI, E. H.; KHAN, M. M.; SHAH, S. A. R.; RAZA, S. S.; SHOAIB, M.; ADNAN, M.. Dynamics of Water Quality: Impact Assessment Process for Water Resource Management. Processes, Basel, v.7, n.2, p.1-14, 2019. DOI: https://doi.org/10.3390/pr7020102

BRASIL. Lei n. 9.433, de 08 de janeiro de 1997. Institui a Política Nacional de Recursos Hídricos, cria o Sistema Nacional de Gerenciamento de Recursos Hídricos, regulamenta o inciso XIX do art. 21 da Constituição Federal, e altera o art. 10 da Lei no 8.001, de 13 de março de 1990, que modificou a Lei no 7.990, de 28 de dezembro de 1989. Brasília: DOU, 1997.

CARR, G.; BLÖSCHL, G.; LOUCKS, D. P.. Evaluating participation in water resource management: $A$ review. Water Resources Research, v.48. n.11, 2012. DOI: https://doi.org/10.1029/2011wr011662

CASTELLETTI, A.; SONCINI-SESSA, R.. Bayesian Networks and participatory modelling in water resource management. Environmental Modelling \& Software, v.22. n.8, p.1075-
1088, 2007. DOI:

https://doi.org/10.1016/j.envsoft.2006.06.003

CRUTCHFIELD, J. A.; BIGGS, J. A.; JOHNSON, R. W.. Water resource management: Economic, conservation, and legal views. Journal Water Pollution Control Federation, v.36, n.12, p.1447-1459, 1964.

ESTERHUIZEN, M.; JAGER, L.; JEZEWSKI, W. A.. The All Towns Reconciliation Strategies a partnership between water resource management and water services. Water Science and Technology: Water Supply, v.19, n.1, p.79-87, 2018. DOI: https://doi.org/10.2166/ws.2018.054

FANG, S.; XU, L.; PEI, H.; LIU, Y.; LIU, Z.; ZHU, Y.; ZHANG, H.. An Integrated Approach to Snowmelt Flood Forecasting in Water Resource Management. IEEE Transactions on Industrial Informatics, v.10, n.1, p.548-558, 2014. DOI: https://doi.org/10.1109/tii.2013.2257807

FERNANDES, V.; SAMPAIO, C. A. C.. Problemática ambiental ou problemática socioambiental? A natureza da relação sociedade/meio ambiente. Desenvolvimento e Meio Ambiente, n.18, p.87-94, 2008.

GEISSEN, V.; MOL, H.; KLUMPP, E.; UMLAUF, G.; NADAL, M.; VAN DER PLOEG, M.; RITSEMA, C. J.. Emerging pollutants in the environment: A challenge for water resource management. International Soil and Water Conservation Research, v.3, n.1, p.57-65, 2015. DOI: https://doi.org/10.1016/j.iswcr.2015.03.002

GIARDINO, C.; BRESCIANI, M.; VILLA, P.; MARTINELLI, A. Application of Remote Sensing in Water Resource Management: The Case Study of Lake Trasimeno, Italy. Water Resources Management, v.24, n.14, p.3885-3899, 2010. DOI: https://doi.org/10.1007/s11269-010-9639-3

GIL, A. C.. Métodos e técnicas de pesquisa social. 6 ed. São Paulo: Atlas, 2008.

GUPTA, R.; KUMAR, G.. Scenario planning for water resource management in semi arid zone. Physics and Chemistry of the Earth, v.105, p.290-299, 2018. DOI: https://doi.org/10.1016/j.pce.2017.12.004

HIRSCH, J. E.. An index to quantify an individuals scientific research output. Proceedings of the National Academy of Sciences of the United States of America, v.102, n.46, p.16569-16572, 2005. DOI: https://doi.org/10.1073/pnas.0507655102 
KARR, J. R.. Biological Integrity: A Long-Neglected Aspect of Water Resource Management. Ecological Applications, v.1, n.1, p.66-84, 1991. DOI: https://doi.org/10.2307/1941848

KOSEOGLU, M. A.. Growth and structure of authorship and co-authorship network in the strategic management realm: Evidence from the Strategic Management Journal. Business Research Quarterly, v.19, p.153-170, 2016. DOI: https://doi.org/10.1016/i.brq.2016.02.001

LIU, X.; PAN, Y.; ZHANG, W.; YING, L.; HUANG, W.. Achieve Sustainable Development of Rivers with Water Resource Management - Economic Model of River Chief System in China. Science of The Total Environment, 2019. DOI: https://doi.org/10.1016/j.scitotenv.2019.134657

MASHAZI, T. P.; MOROLE, M. S.; MODLEY, L. S.. Evaluating public perceptions, attitudes and participation in water resource management: The case of an urban township in South Africa. Water Practice and Technology, v.14, n.2, p.726-731, 2019. DOI: https://doi.org/10.2166/wpt.2019.058

MILLER, D. L.; HUGHES, R. M.; KARR, J. R.; LEONARD, P. M.; MOYLE, P. B.; SCHRADER, L. H.; ORTH, D. J.. Regional Applications of an Index of Biotic Integrity for Use in Water Resource Management. Fisheries, v.13, n.5, p.12-20, 1988. DOI: https://doi.org/10.1577/15488446(1988)013<0012:raoaio>2.0.c

MITCHELL, B.; PRIDDLE, C.; SHRUBSOLE, D.; VEALE, B.; WALTERS, D.. Integrated water resource management: lessons from conservation authorities in Ontario, Canada. International Journal of Water Resources Development, v.30, n.3, p.460-474, 2014. DOI: https://doi.org/10.1080/07900627.2013.876328
MYSIAK, J.; GIUPPONI, C.; ROSATO, P.. Towards the development of a decision support system for water resource management. Environmental Modelling \& Software, v.20, n.2, p.203-214, 2005. DOI: https://doi.org/10.1016/j.envsoft.2003.12.019

OLMSTEAD, S. M.. Climate change adaptation and water resource management: A review of the literature. Energy Economics, v.46, p.500-509, 2014. DOI: https://doi.org/10.1016/j.eneco.2013.09.005

SANTANA, R. A.; BEZERRA, S. T. M.; SANTOS, S. M.; COUTINHO, A. P.; COELHO, I. C. L.; PESSOA, R. V. S. Assessing alternatives for meeting water demand: A case study of water resource management in the Brazilian Semiarid region. Utilities Policy, v.61, 2019. DOI: https://doi.org/10.1016/i.j up.2019.100974

VAN ECK, N. J.; WALTMAN, L.. Citation-based clustering of publications using CitNetExplorer and VOSviewer. Scientometrics, v.111, p.1053-1070, 2017. DOI: https://doi.org/10.1007/s11192-017-2300-7

YU, C.; YIN, X.; YANG, Z.; DANG, Z.. Sustainable Water Resource Management of Regulated Rivers under Uncertain Inflow Conditions Using a Noisy Genetic Algorithm. International Journal of Environmental Research and Public Health, v.16, n.5, 2019. DOI: https://doi.org/10.3390/ijerph16050868

ZIERVOGEL, G.; JOHNSTON, P.; MATTHEW, M.; MUKHEIBIR, P.. Using climate information for supporting climate change adaptation in water resource management in South Africa. Climatic Change, v.103, n.3-4, p.537-554, 2010. DOI: https://doi.org/10.1007/s10584-009-9771-3 